\title{
Current locking in magnetically coupled long Josephson junctions
}

\author{
E. Goldobin* \\ Institute of Thin Film and Ion Technology, Research Center Jülich GmbH, D-52425 Jülich, Germany
}

A. V. Ustinov

Physikalisches Institut III, Universität Erlangen-Nürnberg, D-91058, Erlangen, Germany

(Received 16 April 1998; revised manuscript received 9 November 1998)

\begin{abstract}
Critical currents in a system of two magnetically coupled long Josephson junctions are studied numerically, analytically, and experimentally. We show that the switching from static to dynamic state of one junction can trigger switching of the other, and this process leads to current locking. A distinction is made between different critical currents of an individual junction depending on the static or dynamic state of the other junction. Good agreement between the experiment and numerical simulation is found. [S0163-1829(99)07917-5]
\end{abstract}

\section{INTRODUCTION}

Inductively coupled long Josephson junctions (LJJ's) received much attention during the past years. First, the stacks of these junctions are promising for applications as local oscillators in $\mathrm{mm}$ and sub-mm wave band superconductive receivers. ${ }^{1}$ Second, stacked LJJ's serve as a good model system which allows us to study and understand the processes taking place in intrinsic high- $T_{c}$ Josephson junctions. ${ }^{2}$ In general, coupled LJJ's form an interesting nonlinear physical system where both nonlinearity and interaction between subsystems play an important role. As an example, we recall an interesting physical phenomenon such as Cherenkov radiation of plasma waves by fast moving fluxon which has been confirmed recently in experiment and simulation., 3

In the experiment with LJJ's, an important step of the sample characterization is the measurement of its static properties, i.e., a dependence of the critical current $I_{c}$ on magnetic field $H$. The character of $I_{c}(H)$ dependence can give information about the presence of occasional parasitic flux quanta trapped in the LJJ or in the superconducting film in its vicinity. It also provides a technique to evaluate several important parameters of LJJ (e.g., the critical current density $j_{c}$, the magnetic flux penetration field $H_{c 1}$, the effective magnetic thickness $\Lambda$, etc.) from experiment. Therefore understanding of the static properties of coupled LJJ is important.

In experiments with stacked LJJ's several interesting phenomena such as changing of modulation period of $I_{c}(H)$ pattern, ${ }^{5}$ visibility of fluxon modes ${ }^{6}$ on $I_{c}(H)$ dependence, and new effect called "current locking" (CL ${ }^{7-9}$ were found. In spite of numerous experimental observations of CL, we are not aware of any commonly accepted opinion about the origin of this phenomenon. First it was explained as a result of interaction between the junctions (which sounds reasonable) but it was not shown neither analytically nor by simulation how it appears from the inductive coupling model. ${ }^{10}$ The efforts to derive the CL from the static equations did not lead to any success. Nevirkovets and co-workers ${ }^{8,11}$ proposed the idea that CL cannot be explained in the framework of the inductive coupling model ${ }^{10}$ and it is associated with some other coupling mechanism, e.g., supercurrent (Cooper pair) coupling. ${ }^{12}$

In the present work, ${ }^{3}$ we show by means of numerical simulation that experimentally found CL can be obtained and well explained in the framework of the inductive coupling model. ${ }^{10}$ At the same time, CL cannot be derived from purely static equations. Our numerical investigation of two coupled LJJ's also shows that the critical current $I_{c}(H)$ of one LJJ strongly depends on the dynamic state of the other LJJ. This effect leads to different critical current branches on $I_{c}(H)$ dependence of the individual LJJ in a stack, as observed in experiment.

The next section describes the experimental technique and contains a typical dependence measured experimentally where different characteristic regions can be seen. In Sec. III we use analytical approach to obtain as much information as possible about the static properties of the system and, in particular, about the characteristic values of magnetic field. We were not able to explain all system properties using analytical approach based on static equations and, therefore, in Sec. IV we present the results of numerical simulation of $I_{c}(H)$ dependences for different parameters of the system based on the time dependent equations. As in the experiment, several characteristic regions are found and the behavior of the system in each of them is analyzed and explained in the framework of inductive coupling model. Section V concludes the work.

\section{EXPERIMENTAL TECHNIQUE}

Experimentally, we investigated stacked (Nb-Al$\left.\mathrm{AlO}_{x}\right)_{2}-\mathrm{Nb}$ LJJ's made by standard technology. Details about sample fabrication can be found elsewhere. ${ }^{13}$

Depending on the geometry of the sample under investigation, two different measurement configurations are usually distinguished. If the sample has an electrical contact to the middle superconducting electrode [see Fig. 1(a)], the individual dependences of critical currents of LJJ's on external magnetic field $H$ can be measured. While the bias current is passing through the whole structure, the voltage is measured independently on each LJJ. The critical currents, measured in this way, will be denoted as $I_{s}^{A}$ and $I_{s}^{B}$, while the critical currents of the same but uncoupled LJJ's will be denoted as $I_{c}^{A}$ and $I_{c}^{B}$.

If the geometry of the sample does not provide a contact to the middle superconducting electrode, the dependence of critical currents $I_{s}^{A, B}$ on the external magnetic field $H$ is mea- 

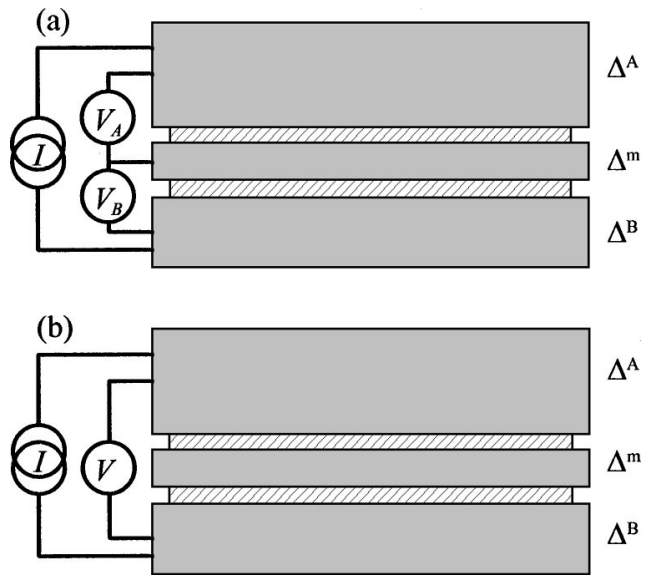

FIG. 1. Two configurations to measure the dependence of critical currents of two coupled LJJ's $I_{s}^{A, B}$ on magnetic field $H$ : (a) if the contact to the middle electrode is provided, the voltage is measured on each LJJ individually; (b) otherwise, one has to measure $I_{c}(H)$ and $I_{g}(H)$ (see Fig. 2). The $\Delta$ 's on the right side denote the energy gaps in different electrodes as discussed in the text.

sured as shown in Fig. 1(b). The current passes through the whole structure and the voltage is measured on two junctions connected in series. In this case, the $I-V$ characteristic (IVC) looks like one shown in Fig. 2. To obtain the value of the critical currents of individual LJJ's, one measures the critical current marked as $I_{c}(H)$ in Fig. 2 and maximum current of the step at $V \approx V_{g}$ marked as $I_{g}(H)$. This step at $V_{g}$ accounts for the state in which one junction is in the superconducting (static) state and another one is switched to the gap voltage $\left(V_{g}\right)$ state. The voltage $V_{g}$ corresponds to the sum of the superconducting energy gaps of two electrodes $V_{g}=\left(\Delta^{A, B}\right.$ $\left.+\Delta^{m}\right) / e$, where $\Delta^{A, B, m}$ is superconducting energy gaps of top, bottom, and middle electrodes, respectively, and $e$ is the electron charge. Here we assume that $\Delta^{A}=\Delta^{B}$ which well corresponds to the experimental situation with thick top and bottom $\mathrm{Nb}$ electrodes. $I_{c}(H)$ and $I_{g}(H)$ account for the critical currents of individual junctions, but in general there is no "one to one" correspondence between $I_{c}, I_{g}$ and $I_{s}^{A}, I_{s}^{B}$. Only in the case of coupled LJJ's with $\Delta^{A} \neq \Delta^{B}$ [e.g., in $\left.\left(\mathrm{Nb}-\mathrm{Al}-\mathrm{AlO}_{x}\right)_{2}-\mathrm{Pb} \mathrm{LJJ} \mathrm{s}\right], V_{g}^{A} \neq V_{g}^{B}$ and therefore one may

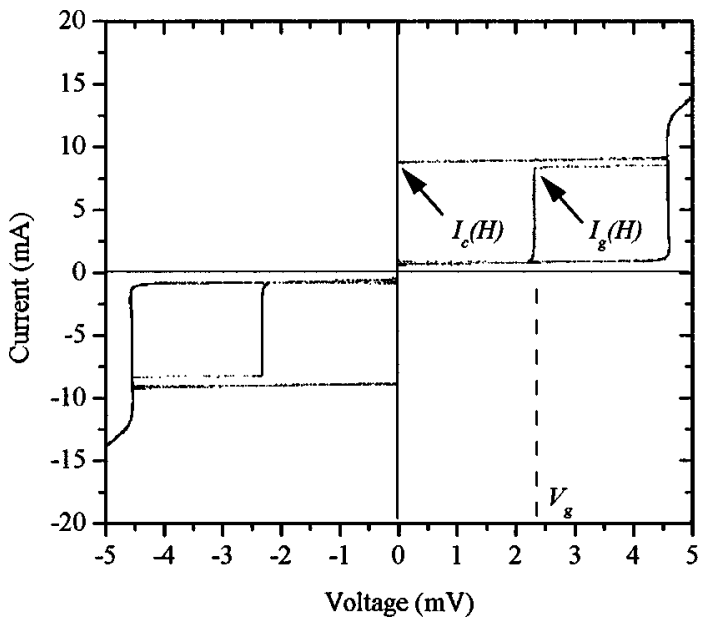

FIG. 2. $I-V$ characteristics of two coupled LJJ's. $I_{c}(H)$ and $I_{g}(H)$ are shown by arrows.

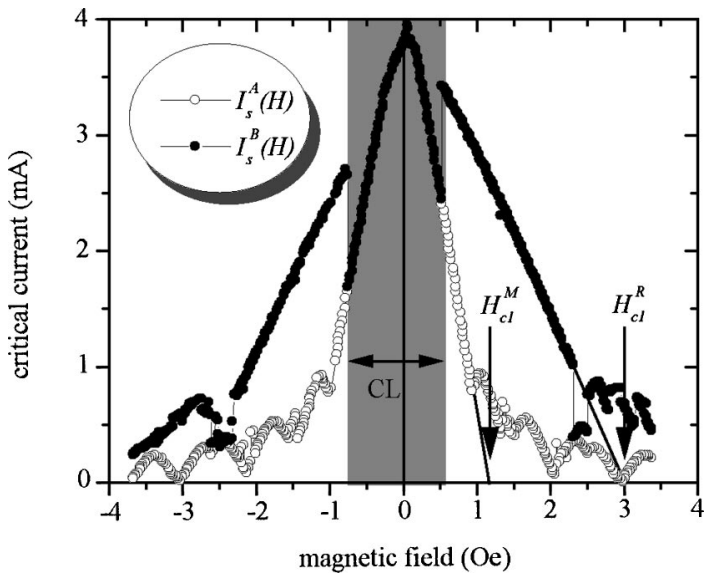

FIG. 3. Experimentally measured dependences $I_{s}^{A, B}(H)$. CL region is clearly visible. At $H \approx 0$ there are few points which do not belong to CL state, in agreement with Fig. 5.

easily find which LJJ switches at $I_{g}$ by measuring $V_{g}$ and comparing it with $V_{g}^{A}$ and $V_{g}^{B}$.

The typical experimentally measured dependence of $I_{s}^{A, B}$ on magnetic field $H$ is shown in Fig. 3. The region of CL is clearly visible in the range $-0.8<H<0.6$ Oe, i.e., increasing the current at given value of field $H$ both LJJ's switch to nonzero voltage state simultaneously. In the CL region the switching of one LJJ induces the switching of the other LJJ. At larger fields junctions switch independently and it is possible to distinguish two characteristic critical fields denoted as $H_{c 1}^{M}$ and $H_{c 1}^{R}$ in Fig. 3. Near the field value of $H$ $\approx 2.4 \mathrm{Oe}$, the $I_{s}^{B}(H)$ curve switches to some other branch with lower critical field. Below we explain these features of the $I_{s}^{A, B}(H)$ dependence in the framework of the inductive coupling model.

\section{SIMPLE ANALYTICAL APPROACH}

The time dependent dynamics of the Josephson phases $\phi^{A, B}$ in two stacked LJJ's is described by a system of two coupled perturbed sine-Gordon equations:

$$
\begin{gathered}
\frac{\phi_{x x}^{A}}{1-S^{2}}-\phi_{t t}^{A}-\sin \phi^{A}=\alpha \phi_{t}^{A}-\gamma+\frac{S \sqrt{D^{\prime}}}{1-S^{2}} \phi_{x x}^{B}, \\
\frac{D^{\prime} \phi_{x x}^{B}}{1-S^{2}}-\phi_{t t}^{B}-\frac{\sin \phi^{B}}{J}=\alpha \phi_{t}^{B}-\gamma+\frac{S \sqrt{D^{\prime}}}{1-S^{2}} \phi_{x x}^{A},
\end{gathered}
$$

where $S$ is the dimensionless coupling constant ${ }^{14,15}(-1$ $<S<0$ ), $D^{\prime}$ is the ratio of effective magnetic thicknesses of $\mathrm{LJJ}^{A}$ and $\mathrm{LJJ}^{B}, J$ is the ratio of the critical current densities $j_{c}^{A} / j_{c}^{B}$. The damping coefficient $\alpha$, normalized bias current $\gamma=j / j_{c}^{A}$ and specific capacitance are supposed to be the same in both LJJ. The coordinate $x$ is normalized to the Josephson penetration depth $\lambda_{J}^{A}$ of uncoupled $\mathrm{LJJ}^{A}$ and the time $t$ is normalized to the inverse plasma frequency $1 / \omega_{p}^{A}$. The definition of dimensionless units can be found in Ref. 16.

The system (1) and (2) should be solved together with boundary conditions

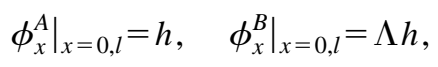

where $\Lambda=\Lambda^{A} / \Lambda^{B}$ is the ratio of effective magnetic thicknesses of the junctions defined in Ref. 16, and $h$ is normal- 

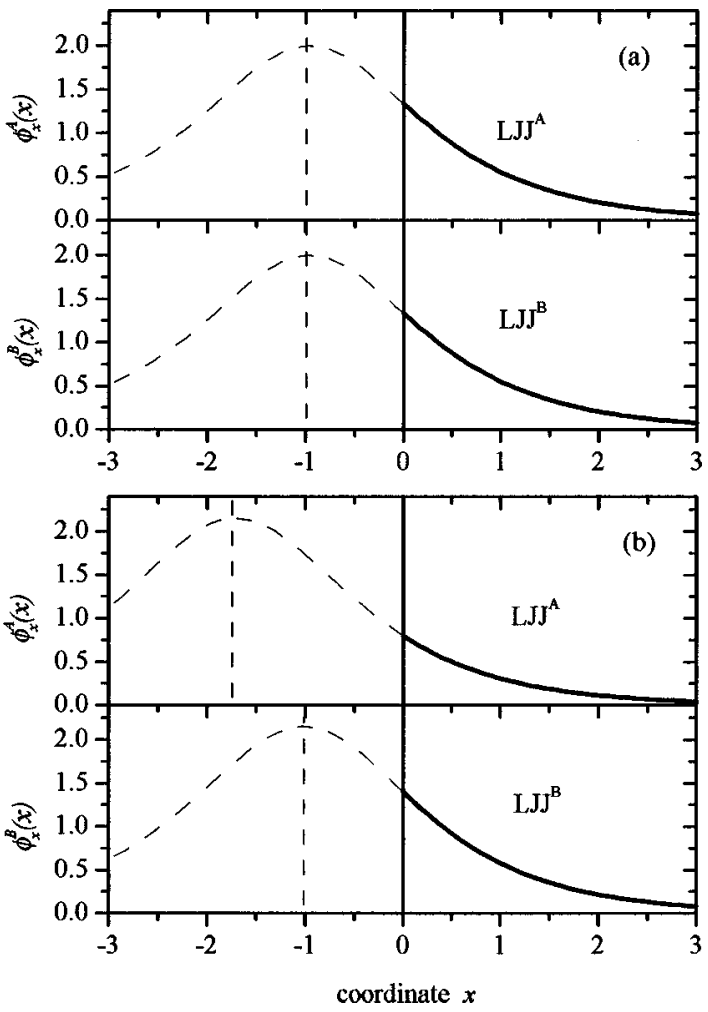

FIG. 4. The penetration of fluxons into coupled LJJ's is shown schematically. (a) equal parameters (symmetric stack); (b) different parameters (asymmetric stack).

ized magnetic field, i.e., $h=2 H / H_{c 1}^{A}$. The first critical field $H_{c 1}^{A}=\Phi_{0} /\left(\pi \Lambda^{A} \lambda_{J}^{A}\right)$ is a field at which fluxon enters the semi-infinite (uncoupled) $\mathrm{LJJ}^{A}$ at $\gamma=0$. It is equal to the field $H_{\text {center }}$ in the center of the static fluxon. The boundary conditions (3) correspond to a stack of overlap geometry placed in the magnetic field $H$.

For the static case, the system of coupled differential Eqs. (1) and (2) with time independent phases $\phi^{A, B}$ can be written in the following simple form:

$$
\begin{gathered}
\frac{\phi_{x x}^{A}}{1-S^{2}}-\sin \phi^{A}=-\gamma+\frac{S \sqrt{D^{\prime}}}{1-S^{2}} \phi_{x x}^{B}, \\
\frac{D^{\prime} \phi_{x x}^{B}}{1-S^{2}}-\frac{\sin \phi^{B}}{J}=-\gamma+\frac{S \sqrt{D^{\prime}}}{1-S^{2}} \phi_{x x}^{A} .
\end{gathered}
$$

\section{A. Symmetric case}

In the case of symmetric stack with identical LJJ's $\left(D^{\prime}\right.$ $=\Lambda=J=1)$ the magnetic field $H$ penetrates completely symmetrically into both LJJ's as shown in Fig. 4(a). We assume that the magnetic field penetrates into each LJJ in the form of single fluxon which is a solution of Eqs. (4) and (5). The center of the fluxon is situated outside the junction in the region $x<0$ in such a way that the boundary conditions (3) are satisfied. In Fig. 4 the profiles $\phi_{x}(x)$ of such fluxons are shown. In the region $x<0$ the profiles are shown by dashed line because it is imaginary part of fluxons and Josephson phase $\phi$ is not defined in this region. In the case of symmet- ric stack, the phases $\phi^{A}(x)=\phi^{B}(x)$ and the system of differential Eqs. (4) and (5) splits into two equivalent uncoupled equations:

$$
\gamma=\sin \phi^{A, B}-\frac{\phi_{x x}^{A, B}}{1+S} .
$$

This equation is the time independent sine-Gordon equation with characteristic length $\lambda_{J}^{+}=1 / \sqrt{1+S}$. Since $S<0$, we note that $\lambda_{J}^{+}>\lambda_{J}=1$ (in normalized units). The renormalization of the Josephson penetration depth results in the change of the first critical field (fluxon penetration field) from $H_{c 1}^{A}$ $=\Phi_{0} /\left(\pi \Lambda^{A} \lambda_{J}^{A}\right)$ to

$$
H_{c 1}^{+}=\frac{\Phi_{0}}{\pi \Lambda^{A} \lambda_{J}^{+}}=H_{c 1}^{A} \sqrt{1+S},
$$

where the superscript " "+, denotes that this quantity is related to the in-phase $\left[\phi^{A}(x)=\phi^{B}(x)\right]$ penetration of fluxons into both LJJ's. In the in-phase case the characteristic size of the fluxon $\lambda_{J}$ increases to $\lambda_{J}^{+}$. Due to the fact that the flux carried by each fluxon is fixed, the field in the center of fluxon decreases from $H_{c 1}^{A}$ to $H_{c 1}^{+}$. Thus the dependence $I_{s}(H)=I_{s}^{A}(H)=I_{s}^{B}(H)$ on external magnetic field in two symmetric LJJ's looks in the same way as that in a single uncoupled LJJ but scaled along the $H$ axis by the factor $\sqrt{1+S}$

The above consideration is valid not in the full range of magnetic fields, but only if $\phi^{A}(x)=\phi^{B}(x)$. This condition is satisfied only for $|H| \leqslant H_{c 1}^{+}$, i.e., when both LJJ's are in the Meissner state. At higher fields, the fluxons penetrate into both LJJ's and form chains that are shifted relative to each other by half of the period. The higher is the density of fluxons, the lower is $\phi_{x x}$. As a result, the coupling term in the equation becomes small and the period of $I_{c}(H)$ becomes nearly equal to that in a single uncoupled LJJ. This problem is considered in detail in Ref. 5.

\section{B. Asymmetric case}

In two asymmetric coupled LJJ's the penetration of fluxons takes place with some shift as shown in Fig. 4(b), therefore $\phi^{A}(x) \neq \phi^{B}(x)$. The system of Eqs. (4) and (5) cannot be reduced then to the single Eq. (6). The boundary conditions (3) become different as well. There are two main reasons for the asymmetry: the difference in the electrode thicknesses which gives $D^{\prime}, \Lambda \neq 1$ and affects the boundary conditions (3) as well as $\lambda_{J}$; and the difference of the critical currents $J \neq 1$, which affects $\lambda_{J}$ and the amplitude $I_{s}^{A, B}(H)$ at $H=0$.

In an asymmetric stack, instead of $H_{c 1}^{+}$, three different critical fields, denoted as $H_{c 1}^{\mathrm{M}}, H_{c 1}^{\mathrm{FF}}$, and $H_{c 1}^{\mathrm{R}}$, can be distinguished $\left(H_{c 1}^{\mathrm{M}}<H_{c 1}^{\mathrm{FF}}<H_{c 1}^{\mathrm{R}}\right)$. $H_{c 1}^{\mathrm{M}}$ corresponds to the penetration of fluxons into one LJJ, while the other LJJ is in the Meissner state. $H_{c 1}^{\mathrm{FF}}$ corresponds to the penetration of fluxons into one LJJ while another LJJ already contains the chain of fluxons moving in the flux-flow state. $H_{c 1}^{\mathrm{R}}$ corresponds to the penetration of fluxons into one LJJ while another LJJ is in the resistive state ( $R$ state). Below, it will be shown that if one of the LJJ's, e.g., $\mathrm{LJJ}^{A}$, is in the $R$ state, the critical 
current of another $\mathrm{LJJ}^{B} I_{s}^{B}=I_{c}^{B}$, i.e., $H_{c 1}^{R}=H_{c 1}^{B}$. The calculation of $H_{c 1}^{\mathrm{FF}}$ for the case when one of the LJJ's contains a chain of fluxons is rather difficult task and it can be accomplished only numerically.

In the previous work, ${ }^{15}$ for the limit of $|S| \ll 1$ and (1 $-\Lambda) \ll 1$, it was derived that

$$
H_{c 1}^{\mathrm{M}} \approx H_{c 1}^{A}\left[\sqrt{1+S}+\frac{3}{2} S\left(\frac{1-\Lambda}{\Lambda}\right)\right]=H_{c 1}^{+}+\Delta H_{c 1} .
$$

From Eq. (8) it it clear that the correction $\Delta H_{c 1}$ caused by the asymmetry is of the second order and therefore is very small. This correction should be neglected in the framework of the first-order perturbation theory. Thus $H_{c 1}^{+}$is a very good approximation for $H_{c 1}^{\mathrm{M}}$ for the majority of the experimentally relevant cases.

\section{NUMERICAL RESULTS}

The critical field in an asymmetric coupled LJJ's has three characteristic values discussed above. Therefore it is interesting to study $I_{s}^{A, B}(H)$ dependences for the fields in the range from 0 to about $H_{c 1}^{\mathrm{R}}$. Since the solution of asymmetric equations cannot be performed analytically, the most straightforward way to study $I_{s}^{A, B}(H)$ dependences is a direct numerical simulation of Eqs. (1) and (2). We take time dependent equations since we would like also to study the dependence $I_{s}(H)$ in one LJJ while the other one is in the dynamic state (e.g., $R$ state). Another reason for this choice is our suspect that CL is related to some time dependent interaction between the LJJ's, since the efforts to derive CL from static equations did not give any results.

The numerical procedure works as follows. For a given set of LJJ's parameters we simulate the IVC of the system at given $h$, i.e., $\bar{V}^{A}(\gamma)$ or $\bar{V}^{B}(\gamma)$ increasing $\gamma$ from zero up. To calculate the voltages $\bar{V}^{A}(\gamma)$ and $\bar{V}^{B}(\gamma)$ in each point of IVC (for each value of $\gamma$ ), we simulate the dynamics of the phases $\phi^{A, B}(x, t)$ by solving Eqs. (1) and (2) together with the boundary conditions (3) numerically using an explicit method [expressing $\phi^{A, B}(t+\Delta t)$ as a function of $\phi^{A, B}(t)$ and $\left.\phi^{A, B}(t-\Delta t)\right]$ treating $\phi_{x x}$ with a five-point, $\phi_{t t}$ with a four-point, and $\phi_{t}$ with a three-point symmetric finite difference scheme. Numerical stability was checked by doubling and dividing in half the spatial and temporal discretization steps $\Delta x$ and $\Delta t$ and checking the influence on the fluxon profiles and on the IVC. The final values used for simulation were $\Delta x=0.025, \Delta t=0.00625$. After simulation of the phase dynamics for $T=20$ time units we calculate the average dc voltages $\bar{V}^{A, B}$ during this time interval as

$$
\bar{V}^{A, B}=\frac{1}{T} \int_{0}^{T} \phi_{t}^{A, B}(t) d t=\frac{\phi^{A, B}(T)-\phi^{A, B}(0)}{T} .
$$

For faster convergence, we use the fact that $\bar{V}^{A, B}$ do not depend on $x$ and therefore we can average the phases $\phi^{A, B}$ in Eq. (9) additionally along the length of the stack.

When the values of $\bar{V}^{A, B}$ are found from Eq. (9), the dynamics of the phases $\phi^{A, B}(x, t)$ is simulated further during $1.2 T$ time units, the dc voltages $\bar{V}^{A, B}$ are calculated for this new time interval and are compared with the previously cal-

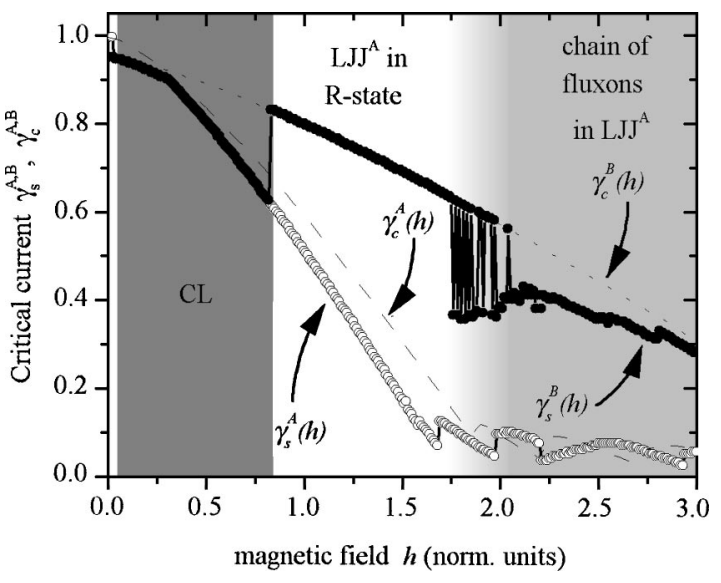

FIG. 5. The dependences $\gamma_{s}^{A, B}(h)$ for two coupled LJJ's at $S$ $=-0.3$ and $\Lambda=2$ obtained numerically. The curves $\gamma_{c}^{A, B}(h)$ obtained for the same uncoupled LJJ's are shown by dashed lines for comparison.

culated values. We repeat such iterations further increasing the time interval by a factor 1.2 until the difference in dc voltages $\left|\bar{V}\left(1.2^{n+1} T\right)-\bar{V}\left(1.2^{n} T\right)\right|$ obtained in two subsequent iterations will become less than a given accuracy $\delta V$ $=10^{-3}$. The particular value of the factor 1.2 was found to be quite optimal and provides fast convergence as well as more effective averaging of low harmonics on each subsequent step. Very small value of this factor, e.g., 1.01 (we remind that only the values greater than 1 have meaning), can result in very slow convergence in the case when $\phi(t)$ contains harmonics with the period comparable to or larger than $T$. Big values of the factor, e.g., 2 or higher, will consume a lot of CPU time already during the second or third iteration and are not good for practical use.

After the voltage averaging for current $\gamma$ is complete, the current $\gamma$ is increased by a small amount $\delta \gamma=0.005$ to calculate the voltages in the next point of the IVC. We use a distribution of phases (and its derivative) achieved in the previous point of the IVC as an initial distribution for the current point. At some current $\gamma$, the LJJ for which we simulate $I_{c, s}(H)$ switches to the nonzero voltage state. The criterion for nonzero voltage is $\bar{V}>0.05$. As it happens, we consider $\gamma$ in the previous point of the IVC as a critical current for a given field $h$. Then the field $h$ is increased by a small amount $\delta h=0.01$ and the IVC is calculated for this new value of $h$ until the critical current $\gamma_{s, c}^{A, B}(h)$ is found.

The difference between LJJ's can be accounted by two parameters: the ratio of critical currents $J$ and the ratio of effective magnetic thicknesses $\Lambda$ which are functions of the electrode thicknesses. The parameter $D^{\prime}$ is also defined through the electrode thicknesses and therefore cannot be considered as an additional free parameter. The simulation of the dependences $\gamma_{s}^{A, B}(h)=I_{s}^{A, B}(h) / I_{c}^{A}(0)$ (normalized critical current) was performed for $|S|=0,0.1,0.2,0.3,0.5,0.8 ; \Lambda$ $=1,1.1,1.2,1.3,1.5,1.7,2.0,2.5,3.0$ (in total $54 \times 2$ curves), $J$ $=1.05$ and $\alpha=0.1$. The small difference in $j_{c}$ was chosen in order to distinguish the CL region from the region of accidental coincidence of the curves $\gamma_{s}^{A}(h)$ and $\gamma_{s}^{B}(h)$. An example of typical dependences $\gamma_{s}^{A, B}(h)$ is shown in Fig. 5 together with the dependences $\gamma_{c}^{A, B}(h)=I_{c}^{A, B}(h) / I_{c}^{A}(0)$ ob- 

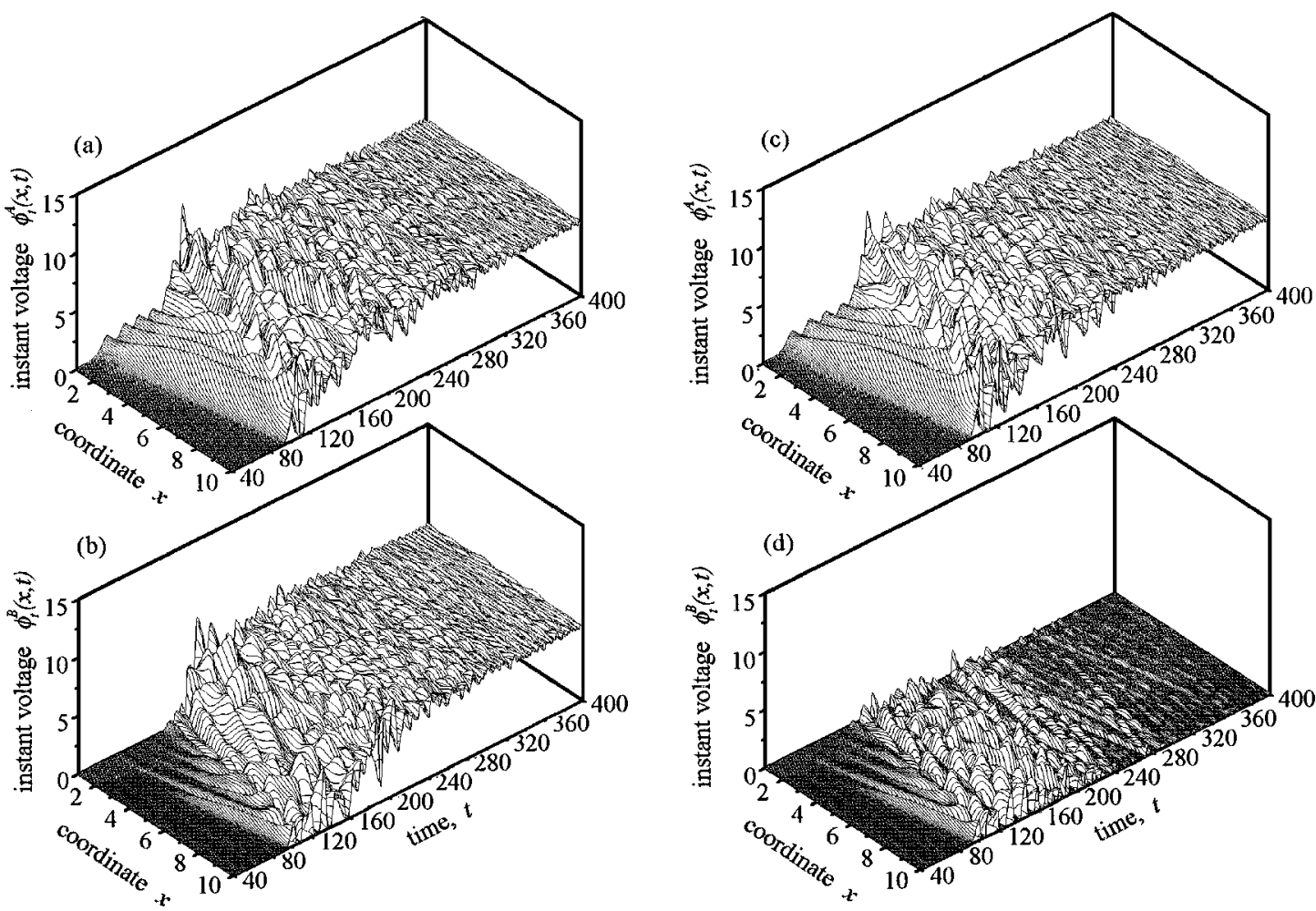

FIG. 6. (a) and (b) show the transient process $\phi_{t}^{A, B}(x, t)$ of switching of both LJJ's (CL) from Meissner state to $R$ state and corresponds to Fig. 5 at $h=0.8$ after increasing of the current from $\gamma=0.635$ to $\gamma=0.640$. (c) and (d) show the transient process of switching of only LJJ ${ }^{A}$ from Meissner state to $R$ state (the absence of CL) and corresponds to Fig. 5 at $h=0.85$ after increasing of the current from $\gamma=0.605$ to $\gamma=0.610$.

tained for $S=0$, i.e., in the same but uncoupled $\mathrm{LJJ}^{A, B}$. The latter two curves are presented for comparison.

One may distinguish several characteristic regions on $\gamma_{s}^{A, B}(h)$ dependences. The first region is very small and appears as a little cusp at $h \approx 0$. In this region the dependences $\gamma_{s}^{A, B}(h)$ follow the behavior of uncoupled $(S=0)$ LJJ's $\gamma_{c}^{A, B}(h)$ and LJJ's switch from the Meissner state independently. We note that this feature reproduces very well the experimental behavior shown in Fig. 3, where a tiny region of independent switching at $H \approx 0$ is also visible.

At finite fields there appears a region of current locking which was observed experimentally in earlier works. ${ }^{8,9}$ In this region, shown by the dark shadow in Fig. 5, LJJ's switch from the Meissner state simultaneously. In CL regime, the LJJ which switches first, triggers the switching of the other LJJ. The fact that at $h=0$ LJJ's switch independently and at higher fields not, is consistent with the inducting coupling model. At $h \approx 0 \phi_{x x}^{A, B}(0) \approx 0$ and, according to Eqs. (1) and (2), LJJ's do not interact. At higher fields $\phi_{x x}^{A, B}(0)$ becomes noticeable, LJJ's interact and may switch simultaneous.

We investigated the profiles of the static phase gradients $\phi_{x}^{A, B}(x)$ (linearly related to the magnetic field profiles) at $h$ $=0.8, \gamma=0.62$ (CL) and $h=0.85, \gamma=0.59$ (no CL) and found no drastic difference. In contrast, the difference is found in the transient switching processes which take place when $\gamma$ exceeds a critical current of one of the LJJ's (LJJ with lower $\gamma_{s}$ at given $h$ ). The transient process for the case of CL is shown in Figs. 6(a) and 6(b) and for the case of no CL in Figs. 6(c) and 6(d). For both values of $h$ mentioned above the transient process starts from the penetration of fluxons into $\mathrm{LJJ}^{A}$. After penetration, the fluxons accelerate up to high velocity $(\alpha \ll 1)$ and induce images of large amplitude in $\mathrm{LJJ}^{B}$, sometimes even creating fluxon-antifluxon pairs in $\mathrm{LJJ}^{B}$. The finite length of LJJ results in the reflection of fluxons (and antifluxons, if any) from the opposite edge of the stack. This transient process looks very chaotic and its result strongly depends on the parameters of the system. In spite of numerous simulations of this transient process for a wide range of the junction parameters, we cannot formulate any criterion which would allow to predict the final state of the transition. In principle, there are three possible final states of each LJJ: Meissner state $(V=0)$, flux-flow state $(V \neq 0)$, and $R$ state $(V \neq 0)$. As a result of the transient process at least one of the LJJ should be at nonzero voltage state. In the case when both LJJ's end up in nonzero voltage state, the CL takes place. In many cases the dependences $\gamma_{s}^{A, B}(h)$ have one large region of CL at low field and, in addition, few smaller domains of CL may appear at higher fields.

In the CL region one may observe a "break" of the curve at some field $h^{*} \approx 0.3$. The value of $h^{*}$ depends on the parameters of the system. For fields $h<h^{*}$, the CL process is driven by $\mathrm{LJJ}^{B}$ (fluxon chains enters into $\mathrm{LJJ}^{B}$ first) while for $h>h^{*} \mathrm{LJJ}^{A}$ drives the CL.

At larger fields $H \sim H_{c 1}^{\mathrm{M}}$, one can see in Fig. 5 that there is again a domain where the junctions switch independently. Note that in this case the first critical field, related to the curve $\gamma_{s}^{A}(h)$, is equal to $H_{c 1}^{\mathrm{M}}$, which corresponds to the situation of simultaneous fluxon penetration into both LJJ's. It is interesting that in this domain $\gamma_{s}^{B}(h)$ exactly coincides with 


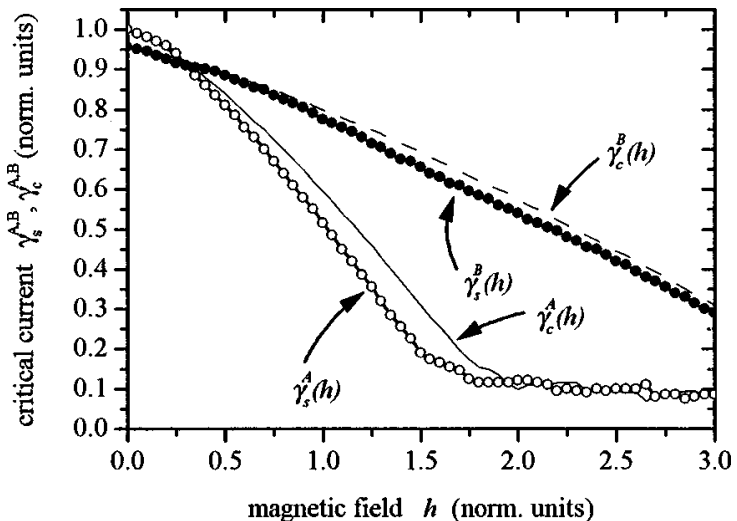

FIG. 7. $\gamma_{s}^{A, B}(h)$ and $\gamma_{c}^{A, B}(h)$ for the same parameters as Fig. 5 but with $\alpha=1$.

$\gamma_{c}^{B}(h)$ (of single uncoupled $\mathrm{LJJ}^{B}$ ), i.e., after $\mathrm{LJJ}^{A}$ switched to the nonzero voltage state, $\mathrm{LJJ}^{B}$ does not "see" $\mathrm{LJJ}^{A}$. Such a behavior can be understood, if one supposes that $\mathrm{LJJ}^{A}$ is in $R$ state. In this case the voltage on the junctions is equal to $V$ $=I R$ and the Josephson phase rotates fast and uniformly, i.e., $\phi(t)=\omega t$, where $\omega=\gamma / \alpha$. In the presence of magnetic field there is an additional phase gradient so that $\phi(x, t)=h x$ $+\omega t$. It is easy to check that such a solution satisfies the sine-Gordon equation without $\sin \phi$ term, i.e., the equation for a superconducting transmission line without Josephson properties. The influence of the term $\sin \phi$ can be easily found using perturbation approach. Looking for a solution in the form

$$
\phi^{A}(x, t)=(h x+\omega t)+A \sin (h x+\omega t)
$$

with $A \ll 1$ and substituting it into the sine-Gordon Eq. (1) without coupling term (we now want to build a solution for single uncoupled LJJ only), we obtain the following expression for the phase in $\mathrm{LJJ}^{A}$ :

$$
\phi^{A}(x, t)=(h x+\omega t)-\frac{1}{\omega^{2}-h^{2}} \sin (h x+\omega t) .
$$

This means that the term describing the influence of $\mathrm{LJJ}^{A}$ on $\mathrm{LJJ}^{B}$ in Eq. (2) is

$$
\phi_{x x}^{A} \propto S \frac{h^{2}}{\omega^{2}-h^{2}} .
$$

Since in conventional tunnel junctions $\omega=\gamma / \alpha \gtrsim 10$ (in experiment $\geq 100$ ), and $h \lesssim 2$, we see that $\omega^{2} \gg h^{2}$ and the term describing the interaction between the junctions vanishes. The real numbers are $\phi_{x x}^{A} \lesssim 10^{-2}$ in simulation and $\phi_{x x}^{A}$ $\$ 10^{-4}$ in experiment.

To check the above idea of decoupling in $R$ state due to low $\alpha$ (and therefore high $\omega$ ) we simulated $\gamma_{c}(h)$ dependence for the system with the same parameters but with $\alpha$ $=1$, in order to have the coupling term (12) of the order of unity. In spite of the fact that such an assumption is unphysical, it will help us to understand the underlying physical mechanisms in the system under question. $\gamma_{s}^{A, B}(h)$ and $\gamma_{c}^{A, B}(h)$ for this case are shown in Fig. 7. From these curves, two conclusions can be made. First, there is no CL here at

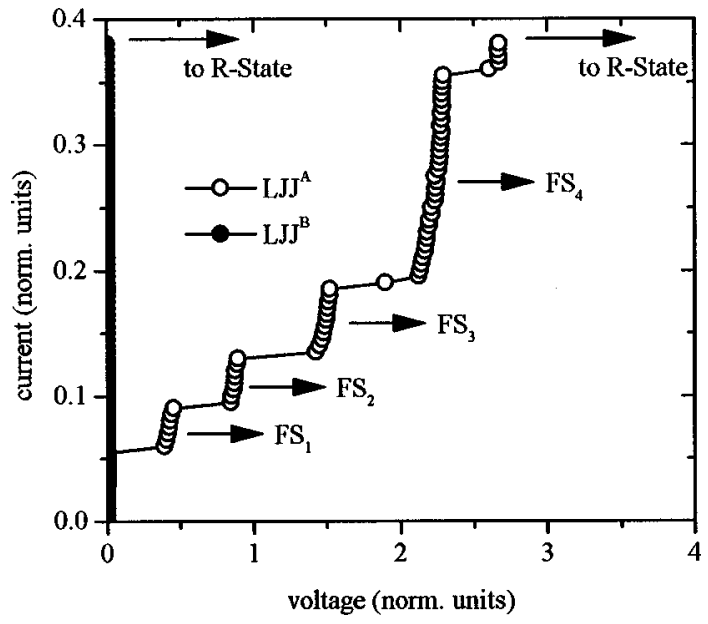

FIG. 8. $I-V$ characteristic at $h=2.0$ with the system parameters corresponding to that in Fig. 5.

all. This is consistent with the consideration given above since in the system with such a high viscosity as $\alpha=1$ the transient process does not develop. This behavior proves that: (a) current locking is a dynamic phenomenon; (b) it cannot be obtained from the static Eqs. (4) and (5) where the dynamics is not taken into account (i.e., $\alpha \gtrsim 1)$. Second, $\gamma_{s}^{B}(H) \neq \gamma_{c}^{B}(H)$. This happens since the coupling term (12) does not vanish and we conclude that the above proposed explanation of the range where $\gamma_{s}^{B}(h)=\gamma_{c}^{B}(h)$ is correct.

Another type of behavior exists at larger fields $H \sim H_{c 1}^{B}$ (light gray area in Fig. 5). In this case the LJJ's switch independently but $\gamma_{s}^{B}(h) \neq \gamma_{c}^{B}(h)$. This happens because $\mathrm{LJJ}^{A}$ is not in the $R$ state, but in flux-flow state, i.e., contains a chain of fluxons moving in flux-flow mode with the velocity smaller than the Swihart velocity. The IVC at $h=2.0$ is
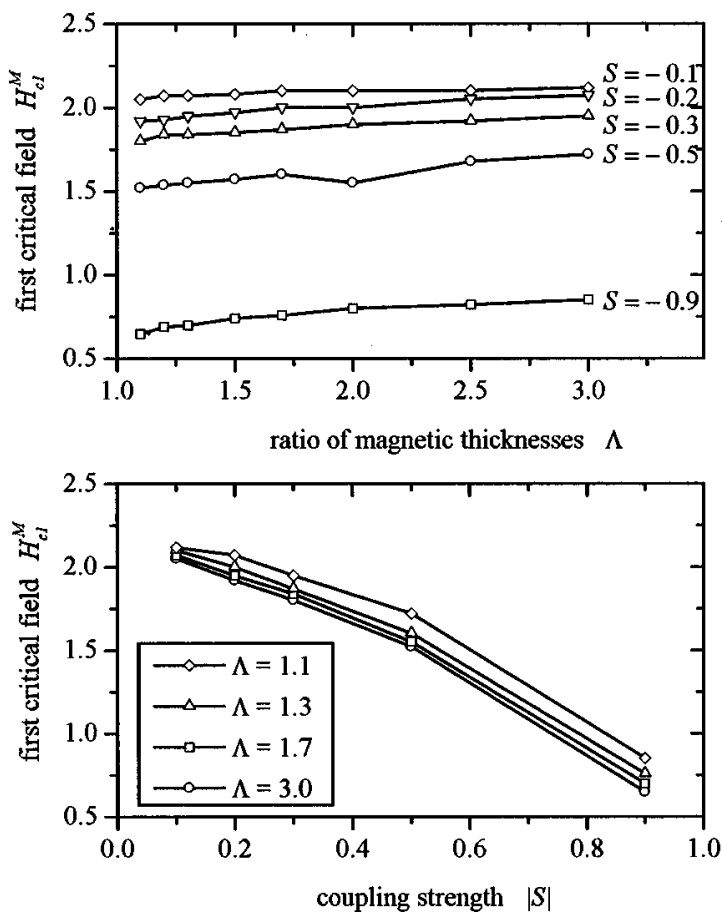

FIG. 9. The dependence of the smallest critical field $H_{c 1}^{M}$ on $|S|$ and $\Lambda$ in the stack of the length $L=20$. 
shown in Fig. 8. One can see that the LJJ ${ }^{A}$ switches to the flux-flow state from the Meissner state. The moving fluxons apparently create a time dependent perturbation of Josephson phase in $\mathrm{LJJ}^{B}$, which results in its switching to nonzero voltage state at lower current than in the case when $\mathrm{LJJ}^{A}$ is in $R$ state.

As we mention in Sec. III, to calculate the values of $H_{c 1}^{M}(\Lambda, S)$ we have to perform a direct numerical simulation. After completing the simulations for different values of $S$ and $\Lambda$, we constructed a table of $H_{c 1}^{\mathrm{M}}(S, \Lambda)$ which is interesting from a practical point of view. The graphs of such dependences are shown in Fig. 9. As one can see, $H_{c 1}^{\mathrm{M}}$ is nearly independent on $\Lambda$ and thus $H_{c 1}^{\mathrm{M}} \approx H_{c 1}^{+}$is a good approximation for experimentally relevant cases.

\section{CONCLUSION}

We studied experimentally, numerically, and analytically the dependences of critical currents of two inductively coupled LJJ's on the applied magnetic field. The behavior of the system in all points of these dependences is well understood. The dependences consist of several regions. The most interesting is the region of the current locking which is a phenomenon characteristic for the nonlinear coupled system under question. We found that current locking can be explained in the framework of the inductive coupling model. ${ }^{10}$ Despite that it appears on the static $I_{c}(H)$ characteristic, cur- rent locking cannot be derived from static equations. It is essentially dynamical phenomenon which occur during the complex transient process of magnetic flux penetration into the junctions. Since the result of the transient process strongly depends on many parameters (due to nonlinear nature of the system), it is very difficult to predict the range of the current locking. CL is an interesting example of the influence of fluxon dynamics on the static properties of coupled LJJ's.

We also found that when one of the LJJ, e.g., $\mathrm{LJJ}^{A}$ is switched into McCumber state, another LJJ follows its $I_{c}(H)$ dependence as it was uncoupled and has a critical field $H_{c 1}^{R}$ $=H_{c 1}^{B}$. In addition, we show that in a system under investigation there are three characteristic magnetic flux penetration fields $H_{c 1}^{\mathrm{M}}, H_{c 1}^{\mathrm{FF}}$, and $H_{c 1}^{\mathrm{R}}$ and we discuss their origin. Comparing numerically calculated values of $H_{c 1}^{\mathrm{M}}$ with analytical expression, we propose a simple approximation $H_{c 1}^{\mathrm{M}} \approx H_{c 1}^{+}$ which is valid for majority of experimentally relevant systems.

\section{ACKNOWLEDGMENTS}

We are grateful to B. A. Malomed for useful discussions and to V. V. Ryazanov for valuable comments. We thank H. Kohlstedt for the sample fabrication. The work was partially supported by the Russian Program for Basic Research.
*Also at Institute of Radio Engineering and Electronics, Moscow, 103907, Russia. Electronic address: gold@ @itech.cplire.ru

${ }^{1}$ V. P. Koshelets, S. V. Shitov, L. V. Filippenko, A. M. Baryshev, H. Golstein, T. de Graauw, W. Luinge, H. Schaeffer, and H. van de Stadt, Appl. Phys. Lett. 68, 1273 (1996).

${ }^{2}$ R. Kleiner, F. Steinmeyer, G. Kunkel, and P. Müller, Phys. Rev. Lett. 68, 2394 (1992); R. Kleiner and P. Müller, Phys. Rev. B 49, 1327 (1994).

${ }^{3}$ E. Goldobin, A. Wallraff, N. Thyssen, and A. V. Ustinov, Phys. Rev. B 57, 130 (1998).

${ }^{4}$ G. Hechtfischer, R. Kleiner, A. V. Ustinov, and P. Müller, Phys. Rev. Lett. 79, 1365 (1997).

${ }^{5}$ S. N. Song, P. R. Auvil, M. Ulmer, and J. B. Ketterson, Phys. Rev. B 53, R6018 (1996).

${ }^{6}$ V. M. Krasnov, N. Mros, A. Yurgens, and D. Winkler, Physica C 304, 172 (1998)

${ }^{7}$ E. Goldobin, H. Kohlstedt, and A. V. Ustinov, Appl. Phys. Lett. 68, 250 (1996)
${ }^{8}$ I. P. Nevirkovets, H. Kohlstedt, and C. Heiden, ICEC Suppl., Cryogenics 32, 583 (1992).

${ }^{9}$ I. P. Nevirkovets, J. E. Evetts, and M. Blamire, Phys. Lett. A 187, 119 (1994).

${ }^{10}$ S. Sakai, P. Bodin, and N. F. Pedersen, J. Appl. Phys. 73, 2411 (1993).

${ }^{11}$ I. P. Nevirkovets (private communication).

${ }^{12}$ I. P. Nevirkovets, J. E. Evetts, M. G. Blamire, Z. H. Barber, and E. Goldobin, Phys. Lett. A 232, 299 (1997).

${ }^{13}$ H. Kohlstedt, A. V. Ustinov, and F. Peter, IEEE Trans. Appl. Supercond. 5, 2939 (1995).

${ }^{14}$ S. Sakai, A. V. Ustinov, H. Kohlstedt, A. Petraglia, and N. F. Pedersen, Phys. Rev. B 50, 12905 (1994).

${ }^{15}$ E. Goldobin, A. Golubov, and A. V. Ustinov, Czech. J. Phys. 46, 663 (1996).

${ }^{16}$ A. Wallraff, E. Goldobin, and A. V. Ustinov, J. Appl. Phys. 80, 6523 (1996). 